\title{
Damage Detection at Welded Joint of Two-Dimensional Plane Model
}

\author{
Chang-Yong Chung, Hee-Chang Eun and Eun-Kyoung Seo \\ Professor, Department of Architecture, Hallym college, Chuncheon \\ Professor, Department of Architectural Eng., Kangwon National Univ., Chuncheon \\ Graduate Student, Department of Architectural Eng., Kangwon National Univ., Chuncheon, Korea
}

http://dx.doi.org/10.5659/AIKAR.2011.13.4.53

\begin{abstract}
Damage detection algorithms based on a one-dimensional beam model can detect damage within a beam span caused by flexure only but cannot detect damage at a joint with prescribed boundary conditions or at the middle part of a beam section where the neutral axis is located. Considering the damage at a welded joint of beam elements in steel structures and modeling the damage with twodimensional plane elements, this study presents a new approach to detecting damage in the depth direction of the joint and beam section. Three damage scenarios at the upper, middle, and lower parts of a welded joint of a rectangular symmetric section are investigated. The damage is detected by evaluating the difference in the receptance magnitude between the undamaged and damaged states. This study also investigates the effect of measurement locations and noise on the capability of the method in detecting damage. The numerical results show the validity of the proposed method in detecting damage at the beam's welded joint.
\end{abstract}

Keywords: Joint, Damage Detection, Mode Shape, FRF, Flexural Performance, Shear Performance

\section{INTRODUCTION}

Structural health monitoring has been receiving a growing amount of interest from researchers in diverse fields of engineering. Many non-destructive methods for improved serviceability and damage detection of structures have been developed with the advent of various kinds of measuring systems.

Structural damage detection techniques involve the process of detecting, locating, and quantifying damage that has occurred in a structure by using the observed changes in the structure response. During the past several decades, a significant amount of research has been conducted in the area of structural damage detection (Doebling, 1998; Pandey, 1991; Sovoz, 2008; Yoon, 2010; Shih, 2009; Rucevskis, 2009; Adewuyi, 2009; Alampalli, 1997; Cruz, 2008).

Corresponding Author: Hee-Chang Eun, Professor Department of Architectural Eng., Kangwon National University Hyoja 2-dong, Chuncheon-si, Gangwon-do, 200-701, Korea

Tel: +82 332506223 e-mail : heechang@kangwon.ac.kr This work was supported by the National Research Foundation of Korea(NRF) grant funded by the Korea government(MEST) (No. 2011-0012164).

This is an Open Access article distributed under the terms of the Creative Commons Attribution Non-Commercial License (http://creativecommons. org/licenses/by-nc/3.0/) which permits unrestricted non-commercial use, distribution, and reproduction in any medium, provided the original work is properly cited.
Damage detection algorithms of a flexural member using a one-dimensional beam element have been well established using flexural curvature and strain characteristics. The change in the modal strain energy before and after the occurrence of damage has been considered as an indicator for detecting and locating the damage. Where a particular vibration mode stores a large amount of strain energy in a particular structural load path, the frequency and shape of that mode are highly sensitive to the changes in that load path caused by the damage.

Kim and Stubbs (1995) applied a damage identification algorithm to locate and size a single crack based on the ratio of modal strain energy of elements before and after the damage. The curvature required for this calculation is commonly extracted from the measured displacement mode shapes using a central difference approximation. Adewuyi et al. (2009) showed that the distributed strain measurements are a more efficient choice than traditional measurement techniques.

Cornwell and Farrar (1999) presented an analytical method based on the changes in the strain energy of the structure. They provided a generalized method that extended from the onedimensional curvature method of beam-like structures to the twodimensional method of plate-like structures. Kim et al. (2003) presented a damage index algorithm to localize and estimate the severity of damage from monitoring changes in modal strain energy. Shi et al. (1998) provided a method to detect the location and to estimate the magnitude of the damage in a structure. This method was carried out in three stages: expansion of measured mode shapes, localization of the damage domain using energy quotient difference, and damage quantification based on sensitivity 
of the modal frequency. Peterson et al. (2001) worked on the damage localization algorithm and used the changes in the modal strain energy between the mode shapes of a calibrated model and the experimentally obtained mode shapes for a timber beam. Shi et al. (2000) proposed a structural damage detection method based on the change of the modal strain energy before and after the damage and its sensitivity as a function of the analytical mode shape changes and the stiffness matrix. Rahmatalla and Eun (2010) presented a damage detection method based on the distribution of constraint forces in the satisfaction of the displacements expanded from measured strain data. While the research in the area of structural damage detection is intensive, there have been few research papers evaluating the performance of the end support of structures with boundary conditions that include damage.

Traditionally, damage detection methods based on flexural performance are utilized in detecting damage within a beam structure itself under the assumption of prescribed end conditions. The structural joint has a weak performance, due to stress concentration and discontinuity of load flow, and therefore is prone to deterioration by the external effects rather than by a member itself. Numerical experiment and design rarely can define the exact boundary conditions in spite of the existence of damage at the joint or end supports, and the central difference approximation of the flexural performance yields abruptly changed curvature at the end support. It is obvious that the damage at the joint is not detectable with the traditional approaches, and thus it is necessary to explicitly establish the boundary condition when detecting the performance at the joint.

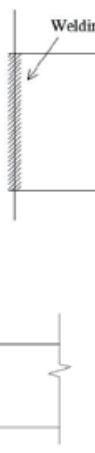

(b)

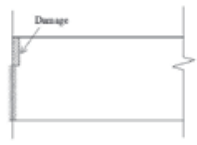

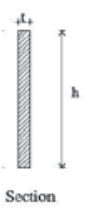

(a)

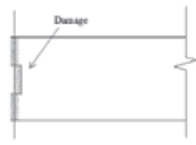

(c)

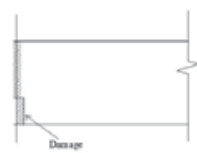

(d)
Figure 1. Damage at beam joint; (a) undamaged joint, (b) damage at the upper part, (c) damage at the middle depth, (d) damage at the lower part

This study models the beam element at the joint as twodimensional finite elements as shown in Fig. 1 (a) and investigates the effect of the performance deterioration due to the damage at the upper, middle, and lower part of the joint as shown in Figs. $1(b),(c)$, and (d), respectively. The proposed method is performed by evaluating the difference of receptance magnitude in the FRFs between the undamaged and damaged states. Considering that the measurement cannot be taken on the damage region itself, this study discusses the measurement ranges within which the damage can be regarded as detectable. The study also considers the effect of the noise contaminated in the measured data on the capability of the proposed method in detecting the damage. The observation of this numerical experiment will provide some fundamental information in detecting the damage at the joint.

\section{FORMULATION}

\subsection{TWO-DIMENSIONAL PLANE STRESS}

The plane stress state of a structure is described by two normal stresses $\sigma_{x}(x, y)$ and $\sigma_{y}(x, y)$, and a shear stress $\tau_{x y}(x, y)$. The Hooke's law for plane stress can be written as:

$$
\left[\begin{array}{c}
\sigma_{x} \\
\sigma_{y} \\
\tau_{x y}
\end{array}\right]=\frac{E}{1-v^{2}}\left[\begin{array}{ccc}
1 & v & 0 \\
v & 1 & 0 \\
0 & 0 & 1-v
\end{array}\right]\left[\begin{array}{c}
\varepsilon_{x} \\
\varepsilon_{y} \\
\gamma_{x y}
\end{array}\right]
$$

where $E$ denotes Young's modulus, $v$ is Poisson's ratio, $E / 2(1+v)=G$, and $G$ is shear modulus. The normal strain in the $\mathrm{x}$ and $\mathrm{y}$ directions, $\varepsilon_{x}$ and $\varepsilon_{y}$, and the shear strain $\gamma_{x y}$ are calculated by

$\varepsilon_{x}=\frac{\partial u_{x}}{\partial x}, \varepsilon_{y}=\frac{\partial u_{y}}{\partial y}, \gamma_{x y}=\frac{\partial u_{x}}{\partial y}+\frac{\partial u_{y}}{\partial x}$

Here, $u_{x}$ and $u_{y}$ represent the displacements in the $x$ and $y$ directions.

Consider a cantilever beam, with length $L$, height $2 a$, and out-ofplane thickness $b$, as shown in Fig. 2. The beam is made from an isotropic linear solid. The right end is clamped and the left end is subjected to a concentrated force $P$. Assuming that $b<<a$, a state of plane stress is considered in the beam. An approximate solution to the stress can be calculated from the Airy function

$$
\phi(x, y)=-\frac{3 P}{4 a b} x y+\frac{P}{4 a^{3} b} x y^{3}
$$

The stresses are developed as

$$
\begin{gathered}
\sigma_{x}=\frac{\partial^{2} \phi}{\partial y^{2}}=\frac{3 P}{2 a^{3} b} x y, \quad \sigma_{y}=\frac{\partial^{2} \phi}{\partial x^{2}}=0 \\
\tau_{x y}=-\frac{\partial^{2} \phi}{\partial x \partial y}=\frac{3 P}{4 a b}\left(1-\frac{y^{2}}{a^{2}}\right)
\end{gathered}
$$

It is found from Eqs. (2) and (4) that the strain energy in the transverse direction is related with its shear performance. Using the boundary conditions of $u_{x}=u_{y}=\partial u_{y} / \partial x=0$ at $x=L$ and $y=0$, the displacement field corresponding to the stress distribution can be found as

$$
\begin{gathered}
u_{x}=\frac{3 P}{4 E a^{3} b} x^{2} y-\frac{P}{4 E a^{3} b}(2+v) y^{3}+\frac{3 P}{2 E a^{3} b}(1+v) a^{2} y-\frac{3 P L^{2}}{4 E a^{3} b} y \\
u_{y}=-v \frac{3 P}{4 E a^{3} b} x y^{2}-\frac{P}{4 E a^{3} b} x^{3}+\frac{3 P L^{2}}{4 E a^{3} b} x-\frac{P L^{3}}{2 E a^{3} b}
\end{gathered}
$$

For investigating the displacement and strain distribution using the above equations, consider a special case of a beam of rectangular cross-section with a thickness of $b=30 \mathrm{~mm}$, a depth of $2 a=600 \mathrm{~mm}$, and a length of $L=1000 \mathrm{~mm}$. The Young's modulus is 200Gpa and the Poisson's ratio is 0.3 . Figures $3(\mathrm{a})$ and (b) represent $u_{x}$ and $u_{y}$ according to the axial $(x)$ and depth $(y)$ directions of the beam. The distribution of the displacement $u_{x}$ in Fig. 3(a) represents the maximum in the top and bottom of the 
section and zero in the neutral axis. The displacement $u_{y}$ is zero at the clamped end and gradually increases in the longitudinal direction of the free end from the clamped end. Figure 3(c) exhibits the distribution of the strain $\varepsilon_{y}$. It showed the maximum strain at the top and bottom of the section of the fixed end and zero strain at the free end. The shear strain in Fig. 3(d) displays the maximum at the mid-depth of the section and minimum at its top and bottom.

The displacements in the axial and depth directions are utilized for calculating the corresponding normal strains of flexural performance as well as the shear strain of shear performance. It is observed that the damage in the depth direction is deeply related to the flexural and/or shear deformation capacity. Thus, a twodimensional model can detect the flexural damage as well as the shear damage.

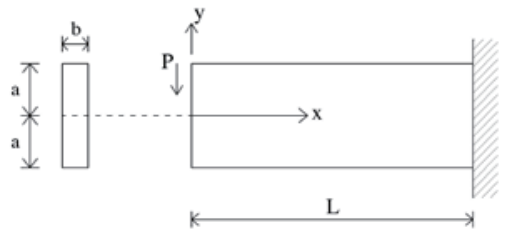

Figure 2. A cantilevered beam

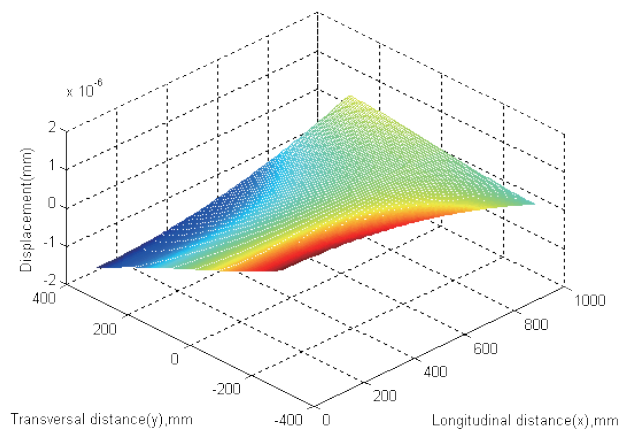

(a)

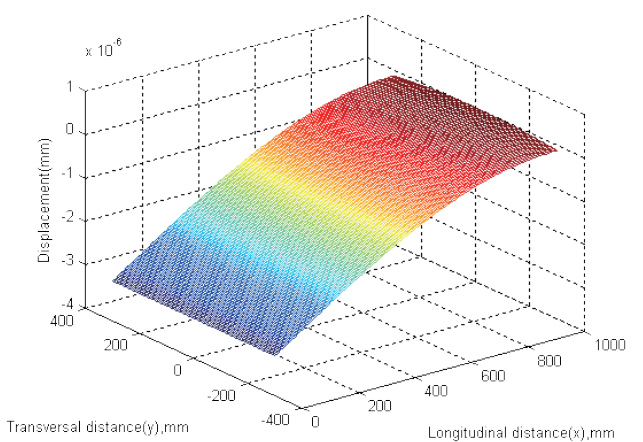

(b)

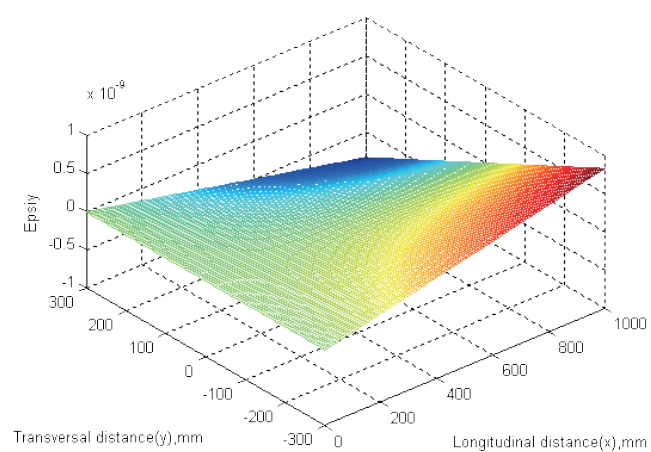

(c)

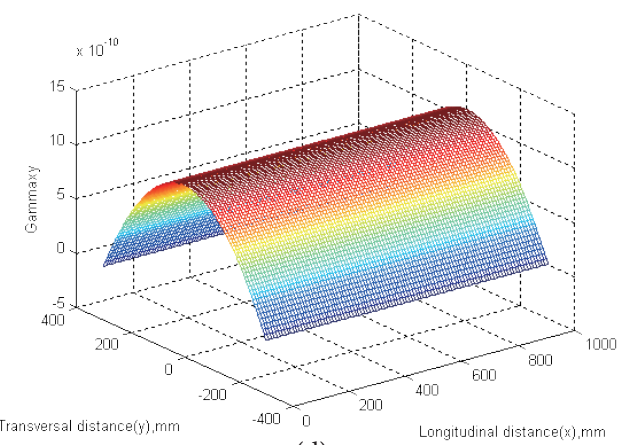

(d)

Figure 3. Plane stress element: (a) distribution of $u_{x}$, (b) distribution of $u_{y}$, (c) distribution of $\varepsilon_{y}$, (d) distribution of $\gamma_{x y}$

\subsection{FINITE ELEMENT FORMULATION}

In this work, the end joint of the beam is modeled by twodimensional elements in the axial and depth directions of the section. Each node of the beam model has only horizontal and vertical displacements as nodal degrees of freedom and no slope as nodal degrees of freedom. The inertia force is included in the equations of equilibrium. These types of beam elements can be applied at the joint of the beam and other members using discretization along the depth as well as the axial direction.

The dynamic equations of motion for the two-dimensional beam are

$$
\begin{aligned}
& \rho \frac{\partial^{2} u_{x}}{\partial t^{2}}=\frac{\partial \sigma_{x}}{\partial x}+\frac{\partial \tau_{x y}}{\partial y}+f_{x} \\
& \rho \frac{\partial^{2} u_{y}}{\partial t^{2}}=\frac{\partial \tau_{x y}}{\partial x}+\frac{\partial \sigma_{y}}{\partial y}+f_{y}
\end{aligned}
$$

where $t$ indicates time, $\rho$ is the mass density, and $f_{x}$ and $f_{y}$ are body forces per unit volume in the $x$ and $y$ directions, respectively. The finite element formulation for the dynamic problem of each beam element is obtained and its combination leads to a dynamic equation of the beam system to be considered.

Using Rayleigh's damping, the dynamic behavior of a structure that is assumed to be linear and approximately discretized for $s(s=2 n)$ degrees of freedom can be described by the equations of motion as

$$
\mathbf{M u ̈}+\mathbf{C} \dot{\mathbf{u}}+\mathbf{K u}=\mathbf{f}(t)
$$

where $\mathbf{M}, \mathbf{C}$ and $\mathbf{K}$ denote the $2 n \times 2 n$ analytical mass, damping and stiffness matrices respectively, $n$ indicates the total number of nodes in the beam system, $\mathbf{u}=\left[\begin{array}{lllll}u_{1 x} & u_{1 y} & u_{2 x} & \cdots & u_{n y}\end{array}\right]^{T}$ , and $\mathbf{f}(t)$ is the $2 n \times 1$ excitation vector. Dynamic responses can be expressed in the time domain and frequency domain. For linear systems there is little loss of information going from the time domain to the frequency domain. In the frequency domain, the FRFs are measured directly from the system instead of measuring the displacement and the force individually. In the time-domain each component is described by a mass, damping and stiffness matrix.

The dynamic characteristics of the beam system can be investigated by the modal data of the natural frequency and its corresponding mode shape or by its corresponding FRFs data. The 
relationships between the FRF and the modal parameters should be established for a successful modal testing. Inserting $\mathbf{u}=\mathbf{U} e^{j \Omega t}$ and $\mathbf{f}=\mathbf{F} e^{j \Omega t}$ into Eq. (7) and expressing it in the frequency domain, it follows that

$$
\left(\mathbf{K}-\Omega^{2} \mathbf{M}+j \Omega \mathbf{C}\right) \mathbf{U}(\Omega)=\mathbf{F}(\Omega)
$$

where $j=\sqrt{-1}, \Omega$ denotes the excitation frequency, and $\mathbf{F}(\Omega)=\left[\begin{array}{llll}F_{1} & F_{2} & \cdots & F_{s}\end{array}\right]^{T}$ and $\mathbf{U}(\Omega)=\left[\begin{array}{llll}U_{1} & U_{2} & \cdots & U_{s}\end{array}\right]^{T}$ represent the Fourier transform of the force and response vectors

f and $\mathbf{u}$, respectively. Equation (8) is valid for an excitation frequency $\Omega$. Defining the FRF matrix $\hat{\mathbf{H}}$,

$$
\begin{gathered}
\hat{\mathbf{H}} \equiv\left|\mathbf{K}-\Omega^{2} \mathbf{M}+i \Omega \mathbf{C}\right| \\
\hat{\mathbf{H}} \equiv\left|\mathbf{K}-\Omega^{2} \mathbf{M}+i \Omega \mathbf{C}\right|
\end{gathered}
$$

where

$$
\hat{\mathbf{H}}(\Omega)=\left[\begin{array}{cccc}
\hat{H}_{11} & \hat{H}_{12} & \cdots & \hat{H}_{1 s} \\
\hat{H}_{21} & \hat{H}_{22} & \cdots & \hat{H}_{2 s} \\
\vdots & \vdots & \ddots & \vdots \\
\hat{H}_{s 1} & \hat{H}_{s 2} & \cdots & \hat{H}_{s s}
\end{array}\right]
$$

$\hat{H}_{i j}$ denotes the displacement response measured at location $i$ due to the unit force input at location $j$.

Assuming the initial system is unexpectedly damaged, in this case, the dynamic response in the frequency domain will not satisfy Eq. (8). Based on the changes in the FRF characteristics, this study detects the damage at the beam's joint.

\section{NUMERICAL EXPERIMENT FOR DAMAGE DETECTION AT WELDED JOINT}

\subsection{UNDAMAGED STATE}

Figure 4 depicts the finite element model of a beam welded to a column surface. The beam adjacent to the joint is modeled by 126 nodes and 192 triangular elements. The beam has elastic modulus of $200 \mathrm{GPa}$, Poisson's ratio of 0.3 , total depth of $300 \mathrm{~mm}$ and unit mass per volume of $30 \mathrm{~kg} / \mathrm{mm}$. The beam has a length of $410 \mathrm{~mm}$ measured from the end support and is connected by a welding of $5 \mathrm{~mm}$ thickness to the column surface. The damping matrix was established as being proportional to the stiffness matrix.

Figure 5 represents the displacement modes in the axial (x) and depth (y) directions corresponding to the first natural frequency. In the plots, the numbers inside the figure indicate the distance from the left end joint of the beam in millimeter. The lines represent the mode shape at each section without noise, and the symbol ' $\square$ ' indicates the mode shape data contaminated with 3\% noise at the corresponding section. It is observed in Fig. 5(a) that the $\mathrm{x}$-component of the mode shape describes the flexural mode and exhibits the maximum displacements at the top and bottom of the section and the minimum displacement at the neutral axis at the mid-depth of the section. The flexural modes at each section in the axial direction of the beam take very similar shapes, but their magnitude gradually increases with the increase in the distance from the joint. The y-component of the mode shape in Fig. 5(b) exhibits the maximum at the top and bottom of the section and the minimum in the vicinity of the neutral axis. It is found that the modal deformation in both axial and depth directions increases with the increase in the distance from the joint. As can be seen

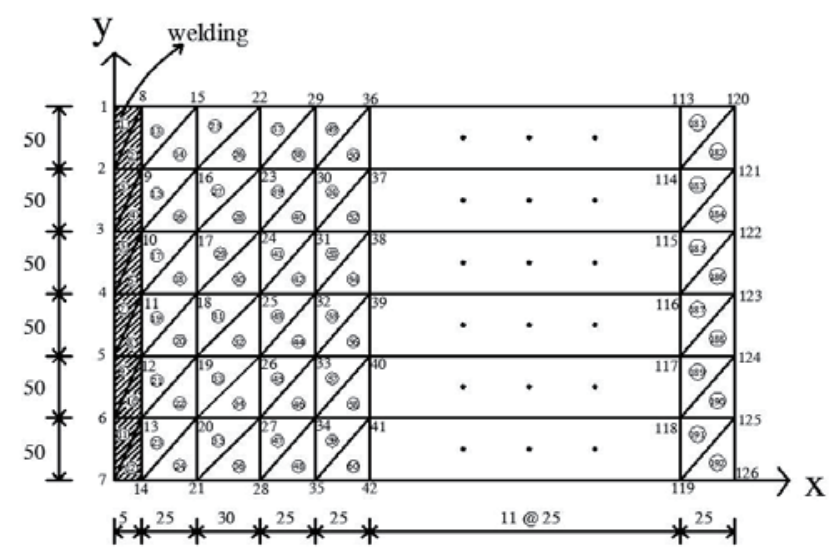

Figure 4. Finite element modeling of the beam in the vicinity of the joint (unit:mm)

from Fig. 5, the 3\% noise rarely affects the mode shape to be obtained numerically.

Figure 6 represents the receptance magnitude at each section in the axial and depth directions of the beam corresponding to the first resonance frequency. Here the receptance magnitude indicates the numerical values measured at all positions due to the impulse in the vertical direction at node 92. Considering that the FRF receptance magnitude takes the absolute value, its $\mathrm{x}$-component in Fig. 6(a) is similar to the corresponding mode shape of Fig. 5(a), where the maximum displacement values were at the top and bottom of the beam and their minimum ones at the mid-depth of the beam section. The difference between the two values of the maximum and minimum magnitudes gradually increases as the

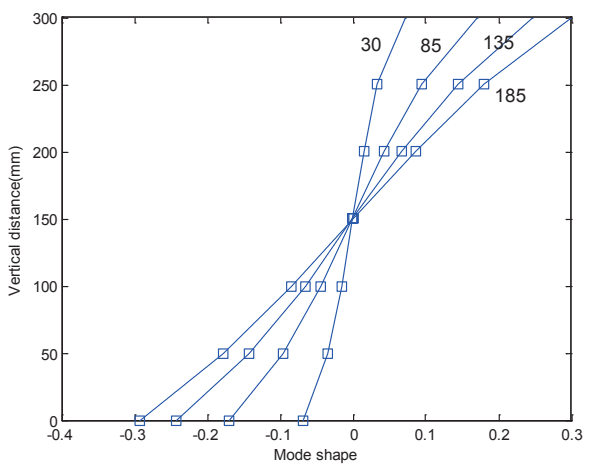

(a)

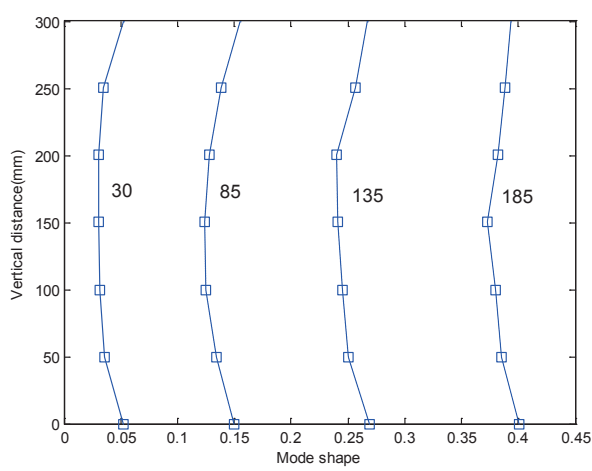

(b)

Figure 5. Mode shape of two-dimensional beam model: (a) x-component, (b) y-component 
distance from the joint increases. The y-component of the FRF in Fig. 6(b) is also similar to that of the mode shape curve of Fig. 5(b), and its magnitude gradually increases with the increase in the distance from the joint. Also, it is observed that the plots are rarely affected by the $3 \%$ noise included in the measured data. In this work, the damage at a part of the beam section will be evaluated and detected by observing the changes in the FRFs before and after the occurrence of the damage.

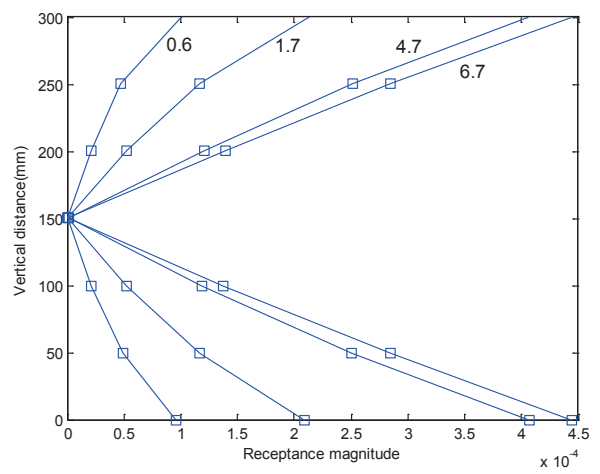

(a)

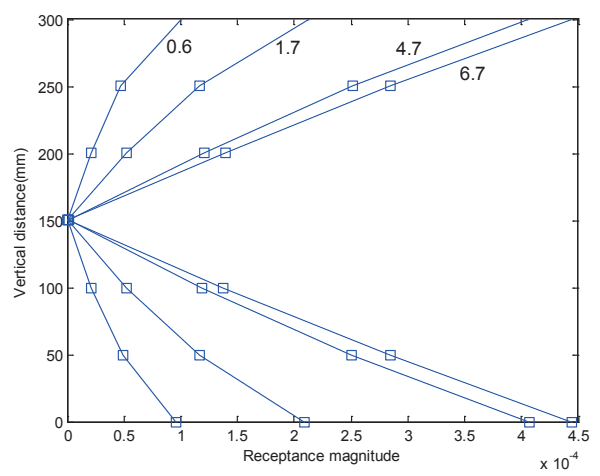

(b)

Figure 6. FRF receptance magnitude of two-dimensional beam model: (a) x-component, (b) y-component

\subsection{DAMAGE STATE}

Consider again the beam in Fig. 4 of Section 3.1 with three damage cases at its welded joint except $E=100 G P a$ and $h=$ $300 \mathrm{~mm}$. The measurement was carried out beyond $6 \mathrm{~mm}$ from the beam end in order to avoid the coincidence of measurement and damage locations. Case 1, Case 2 and Case 3 are considered with damage of $30 \%$ stiffness loss due to poor welding or performance deterioration of the joint, as shown in Figs. 7(a)-(c), respectively. Case 1 has damage at elements 1,2,3 and 4; Case 2 has damage at elements 5, 6,7 and 8; and Case 3 has damage at elements 9, 10, 11 and 12. This work utilized the receptance magnitude measured at all nodes due to the impact in the vertical direction at node 92 on the top surface of the beam. Figure 8 shows the difference in the receptance magnitude between the damaged and undamaged states corresponding to the first resonance frequency of the undamaged state.

The difference of the receptance magnitude is defined as $\left|\left(H_{i, 184}\right)_{u}\right|-\left|\left(H_{i, 184}\right)_{d}\right|, \quad i=1, \quad 2, \quad \cdots \quad 252$

where the subscripts $u$ and $d$ represent the undamaged and damaged states, respectively, and the number 184 indicates the vertical degree of freedom at node 92

Figures 8(a)-(d) represent the difference of the receptance magnitude between the two states at distance $17 \mathrm{~mm}$ from the joint. The difference in the plots enunciate that the damage exists somewhere in the model. Figure 8(a) represents the $\mathrm{x}$-component of the difference of the receptance magnitude of Cases 1 and 3 . The plot exhibits the minimum value in the vicinity of the mid-depth. The differences between the maximum values of Cases 1 and 3 at the top and bottom of the section give an indication of the damage. The behavior in the $y$-component in the difference of the receptance magnitude of Cases 1 and 3, shown in Figure 8(b), has a similar trend to that of the $\mathrm{x}$-component shown in Figure 8(a). Thus it

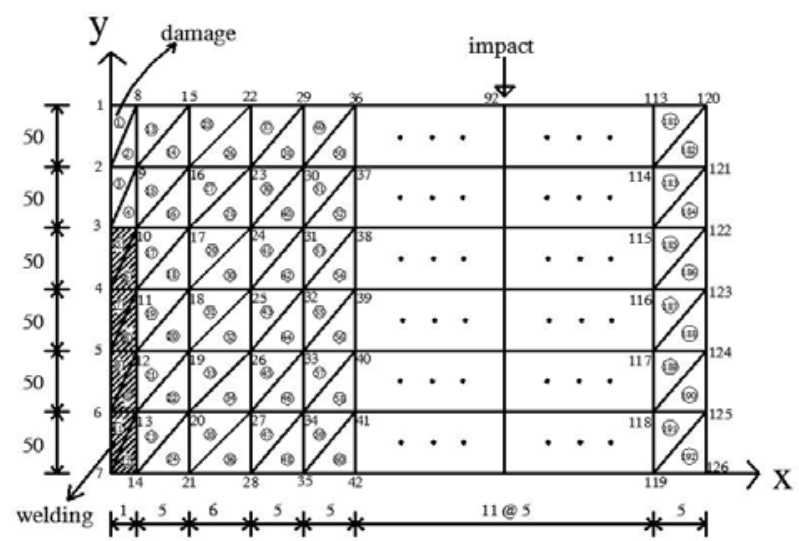

(a)

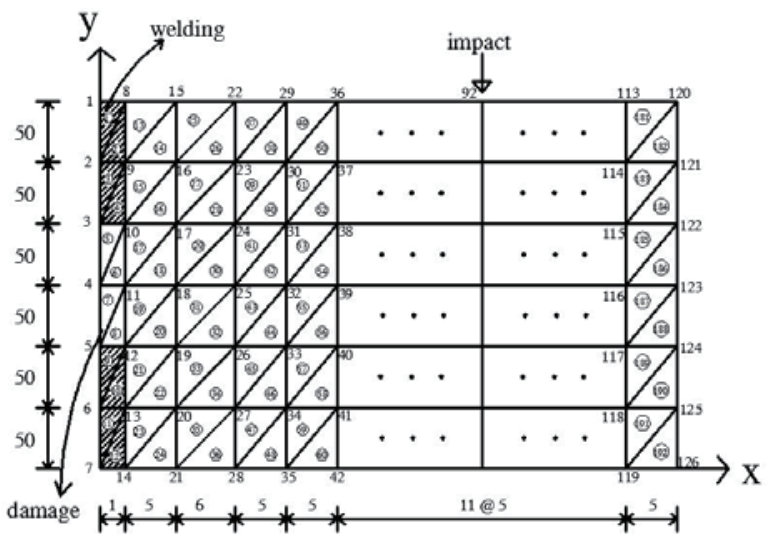

(b)

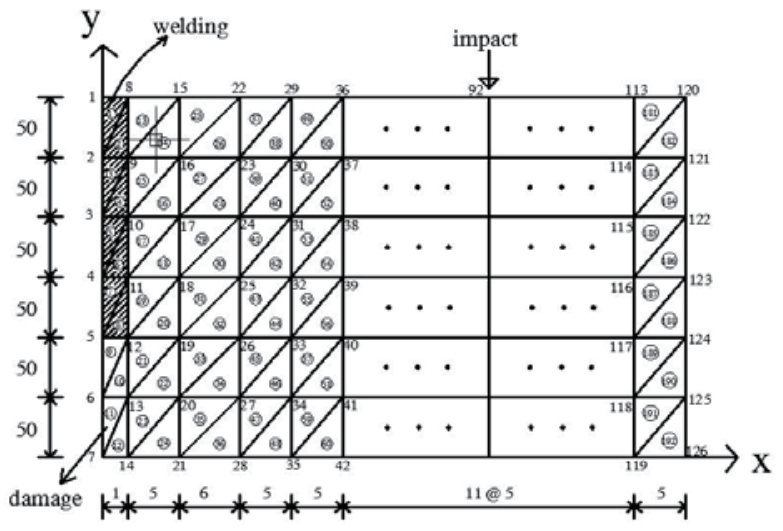

(c)

Figure 7. Finite element modeling modeled by 192 triangular elements adjacent to the joint (unit:mm): (a) Case 1, (b) Case 2, (c) Case 3 
is recognized that the $\mathrm{x}$ - and $\mathrm{y}$-components can be utilized as an index to detect the damage related with the flexural performance in the vicinity of the joint. The $\mathrm{x}$-component of the difference of the receptance magnitude of Case 2, as shown in Fig. 8(c), represents a very small increase in the depth direction compared to those of

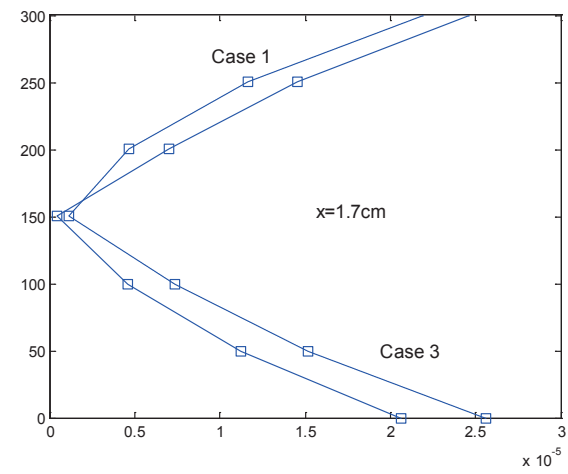

(a)

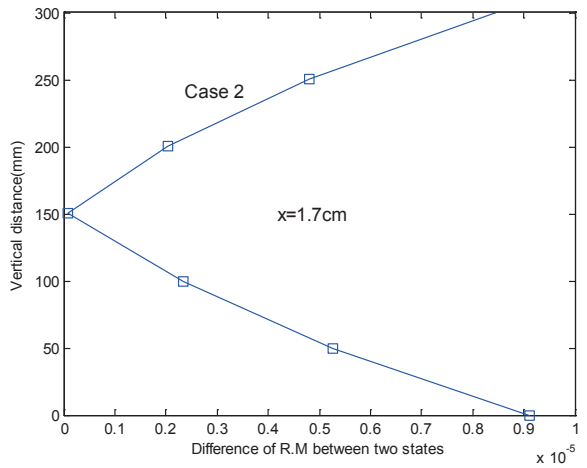

(b)

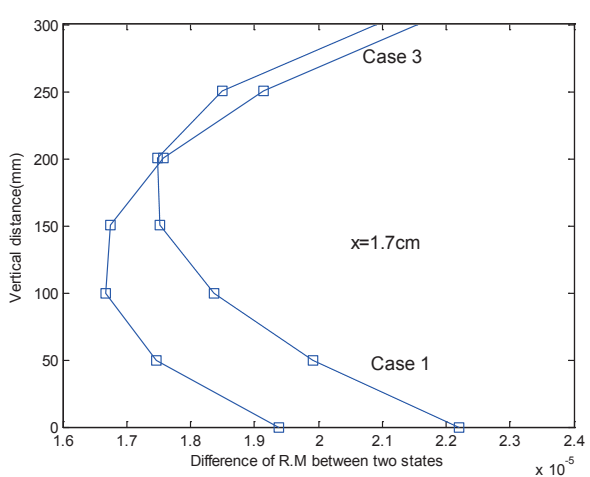

(c)

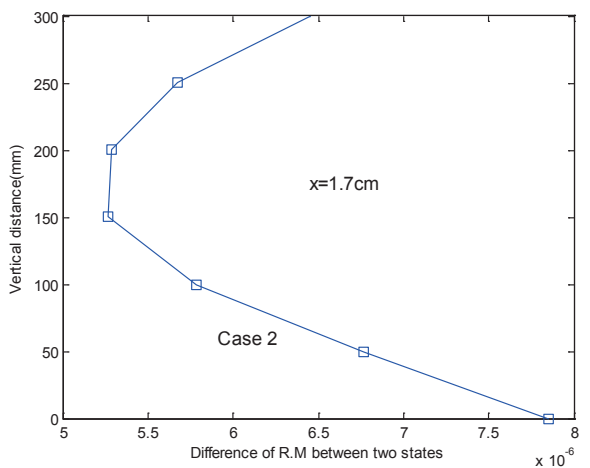

(d)

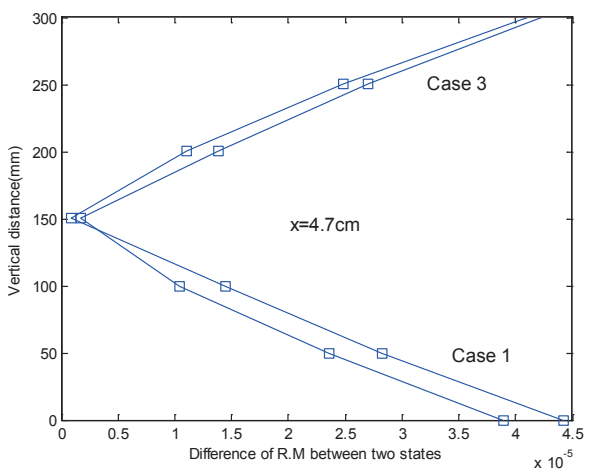

(e)

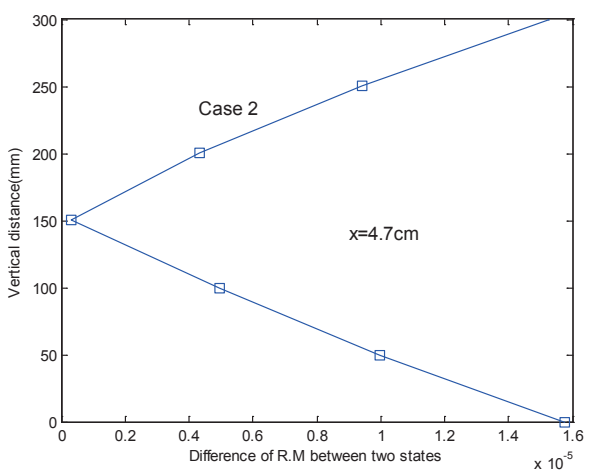

(f)

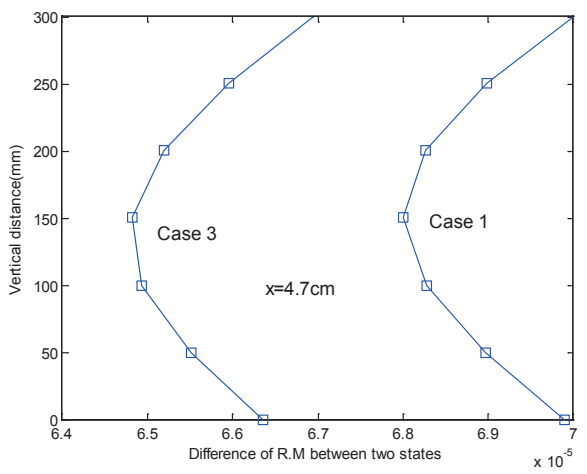

$(\mathrm{g})$

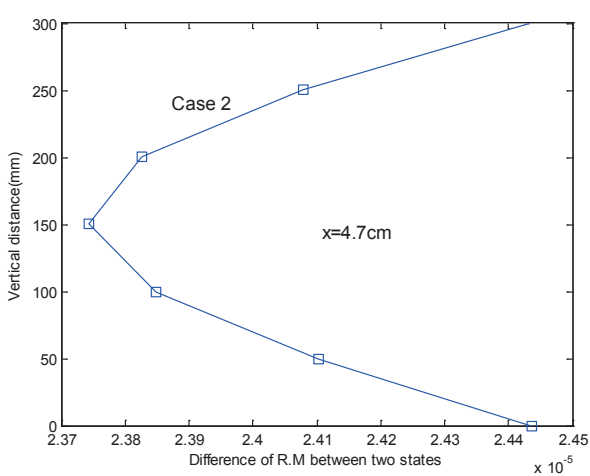

(h)

Figure 8. Receptance magnitude difference between undamaged and damaged states: (a) x-component at location $17 \mathrm{~mm}$ of Cases 1 and 3, (b) $\mathrm{y}$-component at location $17 \mathrm{~mm}$ of Cases 1 and 3, (c) x-component at location $17 \mathrm{~mm}$ of Case 2, (d) y-component at location $17 \mathrm{~mm}$ of Case 2, (e) $\mathrm{x}$-component at location $17 \mathrm{~mm}$ of Cases 1 and 3 , (f) y-component at location $17 \mathrm{~mm}$ of Cases 1 and 3, (g) x-component at location $17 \mathrm{~mm}$ of Case 2, (h) $\mathrm{y}$-component at location $17 \mathrm{~mm}$ of Case 2 
Cases 1 and 3. The small variation implies that any damage does not exist at the upper or lower part of the beam, unlike Cases 1 and 3. The $y$-component of the difference of the receptance magnitude of Case 2 in Fig. 8(d) exhibits the maximum in the vicinity of the mid-depth. Therefore, it is investigated whether the damage in the middle of the beam section is related to the shear performance and is governed by its $\mathrm{y}$-component rather than its $\mathrm{x}$-component. The lines and ' $\square$ ' in the plots represent the ones obtained from measured data without noise and with 3\% noise, respectively.

Again, it is shown that the noise rarely affects the performance of the presented damage detection method.

When checking the difference in the receptance magnitudes in Figs. 8(e)-(h) corresponding to the location $47 \mathrm{~mm}$ from the joint, one can still see the possibility of recognizing the damage at the joint. From the variation of the $\mathrm{x}$-component of the receptance difference in Figs. 8(e) and (f), the damage at Cases 1 and 3 can be explicitly detected at the lower and upper parts, respectively, of the section. The $\mathrm{x}$-component plot of Case 2 in Fig. 8(g) shows a very slight increase compared to Cases 1 and 3 , and it indicates that the damage does not exist at the upper or lower parts of the section as shown in Fig. 8(c). From the observation of Figs. 8(g) and (h) it becomes necessary to investigate the admissible measurement distance from the joint for detecting the damage in the middle of the beam section.

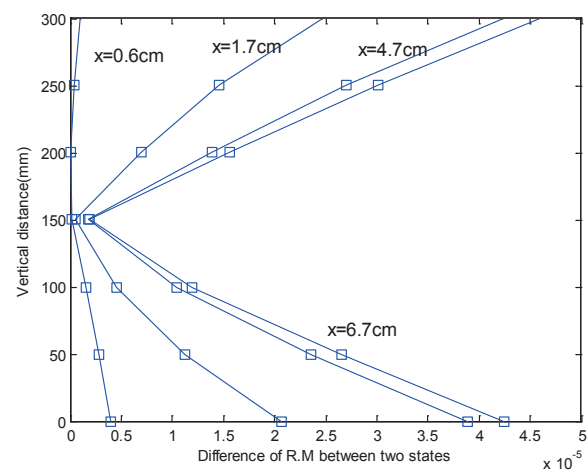

(a)

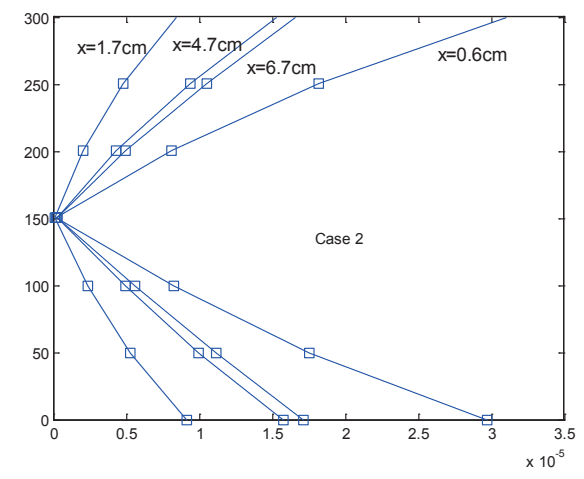

(b)

Figure 9. Receptance magnitude difference between undamaged and damaged states according to the distance from the joint: (a) $\mathrm{x}$-component of Case 3, (b) x-component of Case 2

\subsection{EFFECT OF MEASUREMENT LOCATIONS}

In this section we investigate the regions where the damage can be detected according to the movement of the measurement locations in the axial direction from the joint. Figure 9 displays the $\mathrm{x}$-component of the difference of the receptance magnitude according to the distance from the joint. It is observed from Fig. 9(a) that the damage cannot be detected at lower parts of the beam because of the small differences between the FRF components with the increase in the distance from the end support. But the damage can be detected when the measurements were performed in the neighborhood of the support. Also, the measurement location rarely affects distribution shape regardless of the measurement distance from the joint. And it is observed from Fig. 9(b) that the distribution shape of the $\mathrm{x}$-component takes almost the same shapes regardless of the distance from the joint such that the damage cannot be recognized. It is clear that the damage related to the vertical resisting performance at the joint can be evaluated in the region corresponding to $1 / 6$ of the total depth $50 \mathrm{~mm}$ of the beam. This study showed that structural damage related to the flexural as well as the shear performance at the joint can be detected by measuring the FRFs in the vicinity of the joint.

\section{CONCLUSIONS}

This study presented a new approach to detecting damage at the end joint of flexural beams. The damage at the end joint rarely can be detected using a one-dimensional finite element model because the measured data describe the flexural response under the assumption of prescribed end conditions. Modeling the beam joint as two-dimensional elements, this study investigated methods to detect the damage in the axial and depth directions of the beam section. It has been shown that the damage can be evaluated by investigating the $\mathrm{x}$ - and $\mathrm{y}$-components of the difference of the receptance magnitude of the FRFs before and after the damage. This study also demonstrated the importance of locating the measurements in the vicinity of the joint for better detecting damage related to flexural as well as shear performances. Also, it was shown that the proposed method is rarely affected by external noise.

\section{REFERENCES}

Adewuyi, A.P., Wu, Z. \& Serker, N.H.M.K. (2009) "Assessment of vibration-based damage identification methods using displacement and distributed strain measurements." Structural Health Monitoring, 8: 443-461.

Alampalli, S., Fu, G. \& Dillon, E.W. (1997) "Signal versus noise in damage detection of experimental modal analysis." Journal of Structural Engineering, 123: 237-245.

Cornwell, P., Doebling, S.W. \& Farrar, C.R. (1999) "Application of the strain energy damage detection method to plate-like structures." Journal of Sound and Vibration, 224(2): 359-374.

Cruz, P.J.S. \& Salgado, R. (2008) "Performance of vibration-based damage detection methods in bridges." Computer-Aided Civil And Infrastructure Engineering, 24: 62-79. 
Doebling, S.W., Peterson, L.D., \& Alvin K.F. (1998) "Experimental determination of local structural stiffness by disassembly of measured flexibility matrices." Journal of Vibration and Acoustics, 120: 949-957.

Kim, J.T. \& Stubbs, N. (1995) "Model Uncertainty and Damage Detection Accuracy in Plate-Girder Bridges." Journal of Structural Engineering, 121(10): 1409-17.

Kim, J.T., Ryu, Y.S., Cho, H.M. \& Stubbs, N. (2003) “Damage identification in beam-type structures:frequency-based method vs. mode-shape-based method." Engineering Structures, 25 : 57-67.

Pandey, A.K., Biswas, M. \& Samman M.M. (1991) "Damage detection from changes in curvature mode shapes." Journal of Sound and Vibration, 145: 321-332.

Peterson, S.T., McLean, D.I., Symans, M.D., Pollock, D.G., Cofer, W.F., Emerson, R.N. \& Fridley, K.J. (2001a) "Application of dynamic system identification to timber beams I." Journal of Structural Engineering, 127(40): 418-425.

Peterson, S.T., McLean, D.I., Symans, M.D., Pollock, D.G., Cofer, W.F., Emerson, R.N. \& Fridley, K.J. (2001B) "Application of dynamic system identification to timber beams II." Journal of Structural Engineering, 127(4): 426-432.

Rahmatalla, S. \& Eun, H.C. (2010) "A damage detection approach based on the distribution of constraint forces predicted from measured flexural strain." Smart Materials and Structures, 19 105016

Rucevskis, S., Wesolowski, M. \& Chate, A. (2009) "Vibration-based damage detection in a beam structure with multiple damage locations." Aviation, 13(3): 61-71.

Shi, Z.Y., Law, S.S. \& Zhang, L.M. (1998) "Structural damage localization from modal strain energy change." Journal of Sound and Vibration, 218(5): 825-844.

Shi, Z.Y., Law, S.S. \& Zhang, L.M. (2000b) "Structural damage detection from modal strain energy change." Journal of Engineering Mechanics, 126(12): 1216-1223.

Shih, H.W., Thanbriatnam, D.P. \& Chan, T.H.T. (2009) "Vibration based structural damage detection in flexural members using multi-criteria approach." Journal of Sound and Vibration, 323(3-5): 645-661.

Soyoz, S. \& Feng, M.Q. (2008) "Instantaneous damage detection of bridge structures and experimental verification." Structural Control and Health Monitoring, 15(7): 958-973.

Yoon, M.K., Heider, D., Gillespie, J.W., Ratcliffe, C.P. \& Crane, R.M. (2010) "Local damage detection with the global fitting method using operating deflection shape data." Journal of Nondestructive Evaluation., 29(1): 25-37.

(Date of Submission : 2011.7.15) 